\title{
Campylobacter-Darminfektionen: Optimierung der Labordiagnostik unter Berücksichtigung epidemiologischer Aspekte
}

\author{
Campylobacter bowel infections: optimizing laboratory diagnosis under \\ consideration of epidemiological aspects
}

\author{
Sabine Albert, Sabine Bachmann, V. Brade \\ Abteilung für Medizinische Mikrobiologie im Zentrum der Hygiene, Klinikum der J.W. Goethe-Universität Frankfurt/M.
}

\section{Zusammenfassung:}

Im Zeitraum von 1991 - 1992 wurden 2000 Stuhlproben von a) Tropenreisenden ( $n=$ $600)$, b) Patienten mit Durchfall $(n=500)$ und c) Patienten mit gastrointestinalen Symptomen, jedoch ohne Durchfall $(n=900)$ auf Campylobacter spp. untersucht. Hierfür wurden drei Kultivierungsmethoden parallel eingesetzt: 1. eine Filtermembranmethode, 2. ein blutfreies Campylobacter-Selektivmedium und 3. ein Blutmedium mit ButzlerSupplement. Zusätzlich wurde der Einfluß von Transportbedingungen untersucht mittels Vergleiches zwischen Nativ-Stuhlproben versus Stühlen, die in ein Transportnährmedium (Cary-Blair-Medium) gegeben worden waren $(n=517)$.

Insgesamt waren 54/2000 (2,7\%) Stuhlproben positiv. Die Campylobacter-Auffindungsrate in flüssigen Stühlen (Gruppe B) lag dreifach höher im Vergleich zu nicht-durchfälligen Proben $(p<0.01)$. In den Gruppen b) und c) waren Patienten, die älter als 40 Jahre waren, signifikant seltener mit Campylobacter infiziert als jüngere Personen, während es bei den Tropenreisenden keinen Alterseffekt gab. Unter den drei Kultivierungsmethoden war das Filtermembranverfahren dasjenige mit der besten Campylobacter-Ausbeute $(p<0.01)$ : Methode 1 ergab 81,5\%, Methode $250 \%$ und Methode 3 $40,7 \%$ positive Isolate. Durch den Einsatz von Cary-Blair-Transportmedium konnte die Campylobacter-lsolierungsrate um $25 \%$ gesteigert werden. Die am häufigsten isolierten Spezies waren C. jejuni und C. coli.

\section{Schlüsselwörter:}

Campylobacter - Filtermembranmethode - Selektivmedien - Cary-Blair-Medium Epidemiologie

\section{Summary:}

During 1991 - 1992, 2000 stool specimens from a) foreign travellers $(n=600)$, b) patients with diarrhoea $(n=500)$ and c) patients suffering from gastro-intestinal symptoms without diarrhoea $(n=900)$ were investigated for campylobacter by comparing three cultivation methods: 1. a filter membrane procedure 2. a blood-free medium and 3. a horse-blood medium with Butzler supplement. Additionally, the influence of transport conditions was investigated by comparing nativestool samples versus stools transported in Cary-Blair medium ( $n=517)$

The total campylobacter isolation rate was $54 / 2000(2,7 \%)$. However, the campylobacter isolation rate in diarrhoeal stools (group b) exceeded the isolation rate in nondiarrhoeal samples threefold $(p<0.01)$. Patients older than 40 years in group $b)$ and $c)$ had a significantly lower infection rate with campylobacter than younger patients; however, within the group of foreign travellers there was no age influence on the infection rate. Of the three cultivation methods, the filtration procedure was most successful $(p=<0.01)$ yielding $81,5 \%$ positive results compared to $50 \%$ (method 2 ) and $40,7 \%$ (method 3). The use of Cary-Blair medium increased the isolation rate by $25 \%$. The most frequently isolated species were $C$. jejuni and C. coli.

\section{Keywords:}

Campylobacter - filter membrane procedure - selective media - Cary-Blair medium epidemiology 


\section{Einleitung}

Campylobacter spp. gehören neben Salmonellen weltweit zu den wichtigsten Ursachen von infektiöser Enteritis. Es handelt sich um gramnegative sporenlose bewegliche Bakterien in Kommaform oder regelmäßigen Windungen (1). Die humanpathogenen Arten verursachen überwiegend intestinale Erkrankungen. Die CampylobacterEnteritis gehört zu den Anthropo-Zoonosen: Die meisten Erkrankungen werden durch Verzehr von ungenügend erhitztem Geflügelfleisch und anderen Tierprodukten (z.B. Milch) erworben (2). Klinisch kann die Infektionskrankheit nicht von anderen bakteriell bedingten Diarrhoen unterschieden werden. Nur der Erregernachweis im Stuhl erlaubt eine sichere Diagnose. Nach älteren WHO-Berichten variierte der Prozentsatz der Campylobacter-Isolierung von Patienten mit Diarrhoe abhängig von der geographischen Lage zwischen 2,5\% (Norwegen) und $34 \%$ (Südafrika) (3). Bezogen auf die offiziell gemeldeten Fälle von infektiöser Enteritis wurde in neueren Auswertungen seit Beginn der 80er Jahre für europäische Länder eine Campylobacter-Isolierungsrate zwischen $30-40 \%$ ermittelt $(4,5)$.

In der Veterinärmedizin ist Campylobacter als Sepsisbzw. Durchfallerreger schon seit langem bekannt $\mathrm{m}$ (6-8). Seine Bedeutung in der Humanmedizin als Enteritiserreger ist dahingegen erst seit ca. 20 Jahren unumstritten, nachdem es gelungen war, mittels einer Filtertechnik Campylobacter aus Stuhlkulturen anzuzüchten (9). Alle früheren Versuche einer Isolierung mißlangen, weil nicht bekannt war, daß Campylobacter spp. einerseits eine kapnophile Athmosphäre (mit ca. 5-7\% $\mathrm{O}$ und $7-10 \%$ $\mathrm{CO}_{2}$ ) zum optimalen Kulturwachstum benötigen, zum anderen in den üblichen Stuhlkulturen durch die physiologischen Keime der Darmflora überwuchert werden. Einige Jahre später entwickelte Skirrow ein Campylobacter-Selektiv-Nährmedium mit Zusatz verschiedener Anitbiotika, die das Wachstum der physiologischen Stuhlflora weitgehend hemmen (10). Seitdem ist eine Reihe von weiteren Selektivmedien entwickelt worden (11-15).

In den meisten Teilen der Welt herrscht $C$. jejuni als dominierende Spezies vor (2), aber auch C. coli, C. upsaliensis, C. laridis u.a. werden als Enteritiserreger verantwortlich gemacht $(2,16)$.

Bis zum Beginn dieser Studie wurde in der Abteilung für Medizinische Mikrobiologie, Universitätsklinikum Frankfurt/M. in der Routinediagnostik nur eine CampylobacterSelektiv-Agarplatte eingesetzt. In der vorliegenden Arbeit sollte geprüft werden, ob die bis dahin geringe Campylobacter-Auffindungsrate durch den gleichzeitigen Einsatz von drei Kultivierungsmethoden gesteigert werden kann. Darüber hinaus sollte untersucht werden, ob sich die Campylobacter-Ausbeute durch den Einsatz eines Transportnährmediums erhöhen läßt. Bei der Auswertung sollten zudem auch anamnestische bzw. epidemiologische Aspekte mitberücksichtigt werden.

\section{Material und Methoden}

\section{Probanden/Patienten}

In einem 2-jährigen Zeitraum (1991 - 1992) wurden in der Abteilung für Medizinische Mikrobiologie 2000 Stuhlproben von a) Tropenreisenden $(n=600)$, b) Patienten mit Durchfall (wässrige Stuhl-Konsistenz zum Zeitpunkt der Untersuchung, $n=500$ ) und c) Patienten mit gastrointestinalen Symptomen - jedoch ohne akuten Durchfall (feste Stuhl-Konsistenz zum Zeitpunkt der Untersuchung, $\mathrm{n}=900$ ) auf Campylobacter spezies untersucht. Das Alter lag zwischen 1 Monat und 92 Jahren.

Die Gruppe der Tropenreisenden (a) setzte sich aus Angestellten (und deren Familienangehörigen) der Gesellschaft für Technische Zusammenarbeit (GTZ, Eschborn) zusammen; bei diesem Kollektiv handelte es sich vorzugsweise um symptomlose Probanden, die im Auftrag ihres Arbeitgebers in Ländern der Dritten Welt arbeiten und zwischenzeitlich in Deutschland (Eschborn) medizinischen Routine-Untersuchungen unterzogen wurden. Unter diesen 600 Stuhleinsendungen befanden sich $19(3,2 \%)$ dünnflüssige Proben.

Die Stuhlproben der Patientengruppen b) und c) wurden aus Abteilungen der Universitätsklinik Frankfurt/M. sowie aus umliegenden Krankenhäusern eingesandt.

\section{Transportnährboden (nach 17)}

517 der 2000 Stuhlproben wurden jeweils parallel untersucht: a) Nativ-Stuhlprobe und b) Stuhl, der vor dem Versand in Transportnährmedium (Cary-Blair-Medium, Fa. Unipath/Oxoid CM 519) gegeben wurde.

\section{Kultivierungsmethoden}

1) Filtermembranmethode (nach 18):3-4 Tropfen einer Faecalsuspension (entweder flüssiger Stuhl direkt oder Stuhl in physiologischer $\mathrm{NaCl}$ aufgeschmemmt) wurden auf die Oberfläche eines Membranfilters (Porengröße 0,45 um, Fa. Millipore) gegeben, der zuvor auf eine Mueller-Hinton-Schafsblut-Agrarplatte (Fa. GMN) aufgelegt worden war. Der Filter wurde nach 30-60 min entfernt.

2) Blutfreier Campylobacter-Selektiv-Agar (Fa. Unipath/ Oxoid CM 739) mit Cefoperazon-Selektiv-Supplement (Fa. Unipath/Oxoid SR 125).

3) Campylobacter-Selektiv-Agar auf Blutbasis (Fa. Unipath/Oxoid CM 331), 5-7\% Pferdeblut und ButzlerSupplement (Fa. Unipath/Oxoid SR 085E).

Der Stuhl wurde bei den beiden festen Selektivmedien direkt auf die Platten fraktioniert ausgestrichen.

Die Bebrütung erfolgte mikroaerophil (CampyPak Plus, Fa. Becton Dickinson 71045) für $48-72$ Stunden bei $36^{\circ} \mathrm{C}$. Auf eine Bebrütung bei $42^{\circ} \mathrm{C}$ wurde verzichtet, um gegebenenfalls auch nicht-thermophile Campylobacter-Spezies isolieren zu können. Zudem zeigten Studien von anderen Autoren, daß selbst bei einer Temperaturvon $42^{\circ} \mathrm{C}$ eine Kontamination der Selektivplatten mit Faecalflora (Enterobakteriazeen, Pseudomonaden, Hefen etc.) nicht auszuschließen ist $(15,19)$. Bolton et al. (1988) evaluier- 
ten sogar ein besseres Kulturergebnis hinsichtlich der Campylobacter-Ausbeute bei einer Bebrütungstemperatur von $37^{\circ} \mathrm{C} \mathrm{im} \mathrm{Vergleich} \mathrm{zu} 42{ }^{\circ} \mathrm{C}$ (19).

\section{Speziesdifferenzierung}

Beim Begutachten dẹr Agarplatten wurde überprüft, ob ein typisches Wachstum auf den Nährmedien erfolgt war. Campylobacter spp. wachsen häufig in flachen irregulär begrenzten Kolonien (zerfließendes Wachstum), bzw. die Kolonien gleichen auch kleinen einzelnen Wassertropfen.

Verdächtige Kolonien wurden mittels Phasenkontrastmikroskop auf ihre Morphologie und Beweglichkeit hin untersucht und nach Gram gefärbt; hierbei wurde berücksichtigt, daß ältere Kulturen statt der schraubenförmigen auch kokkoide Formen annehmen können. Zur weiteren Identifizierung wurden die biochemischen $\mathrm{Re}$ aktionen (Oxidase, Katalase, Hippurathydrolyse) sowie die Antibiotikasensitivität mittels Agardiffusionstests mit Cephalotin- und Nalidixinsäureplättchen (jeweils $30 \mathrm{ug}$ ) geprüft (1). In einzelnen Fällen wurde zusätzlich API Campy (Fa. BioMerieux) eingesetzt.

\section{Statistik}

Die statistische Auswertung erfolgte auf der Basis der $x^{2}$. Verfahren. Alle Berechnungen wurden mit Hilfe von SPSS PC, V.3.1 durchgeführt. Für die Alterseffektberechnung erschien eine Aufteilung in engmaschige Alterskategorien wegen der geringen absoluten Gesamtzahl der positiven Befunde ( $n=54)$ nicht sinnvoll. Die Probanden wurden daher in die Altersgruppen 0 - 20 Jahre, 21 - 40 Jahre und > 40 Jahre eingeteilt.

\section{Ergebnisse}

\section{Epidemiologie}

Insgesamt waren $54 / 2000(2,7 \%)$ Stuhlproben positiv, wobei jedoch die Campylobacter-Auffindungsrate in flüssigen Stühlen ca. dreifach höher lag im Vergleich zu nicht-durchfälligen Proben (Abb. 1), was statistisch signifikant ist $\left(x^{2}=15,93, d f=2, p<0.01\right)$. Unter den Patienten mit Durchfall (Gruppe b) befanden sich 5,2\% (26/500) positive Campylobacter-Isolate, während das Kollektiv der Tropenreisenden (Gruppe a) nur in 2,0\% (12/600) und die Patienten ohne Durchfall (Gruppe c) lediglich insgesamt zu 1,77\% (16/900) kulturell positiv waren. Ein signifikanter Unterschied der Tropenreisenden-Gruppe a-im Vergleich zu den Patienten ohne Durchfall - Gruppe c war nicht zu beobachten. Es ist anzumerken, daß unter den 12 Campylobacter-positiven Tropenreisenden 3Probanden durchfällige Stühle aufwiesen.

Die Altersverteilungen der Probanden ließ im Hinblick auf die Campylobacter-Auffindungsrate einen Alterseffekt bei den Patienten mit und ohne Durchfall erkennen, nicht jedoch bei den Tropenreisenden (Abb. 2 - 4). So war beispielsweise die relative Infektionshäufigkeit bei den jüngeren Alterskategorien der Patienten mit Durchfall deutlich erhöht im Vergleich zu den Patienten, welche älter als 40 Jahre waren (Abb. 3). Dieser Unterschied ist statistisch sehr signifikant $\left(x^{2}=13,26 \mathrm{df}=2, p<0.01\right)$. Bei Patienten ohne Durchfall (Gruppe c, Abb. 4) fiel auf, daß

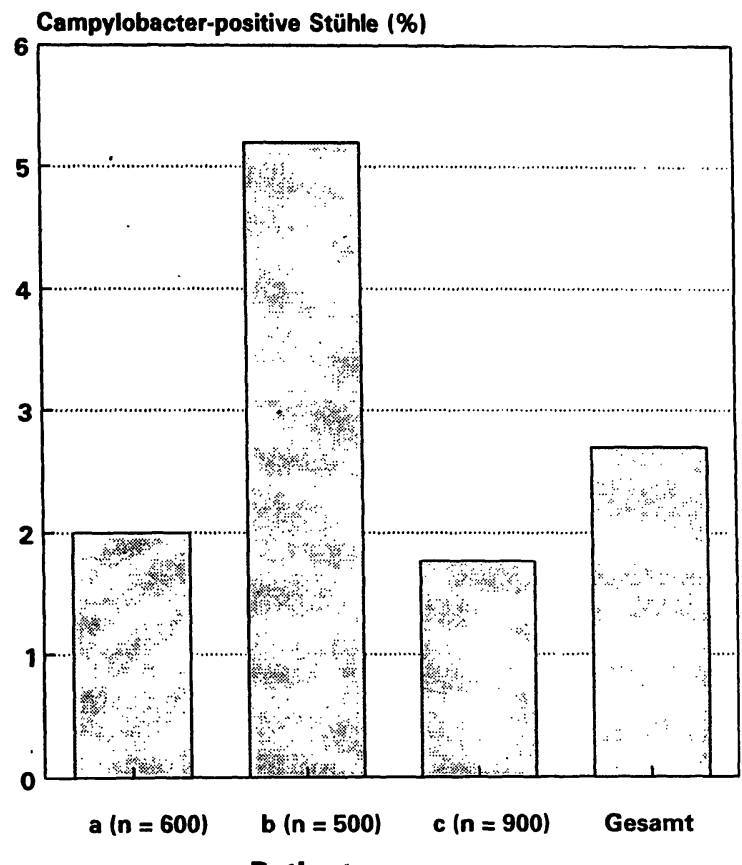

Patientengruppen

Abb. 1: Nachweis von Campylobacter bei drei Patienten- bzw. Probandengruppen $(n=2000)$ a Tropenreisende, $b$ Patienten mit Durchfall, c Patienten mit gastrointestinalen Symptomen, jedoch ohne Durchfall.

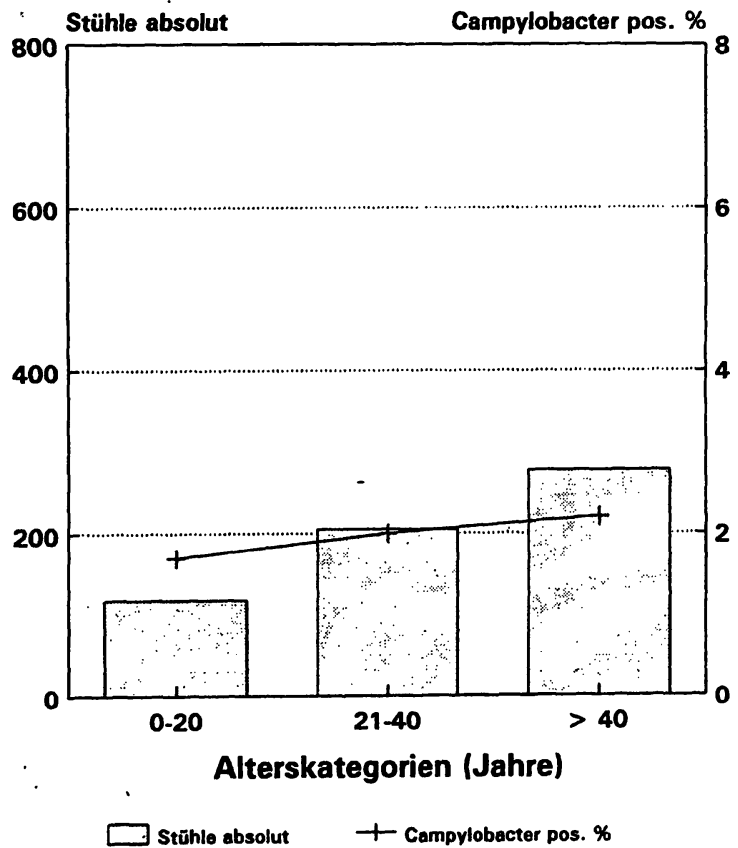

Abb. 2: Nachweis von Campylobacter bei Tropenreisenden (Gruppe $a, n=600$ ) - Altersverteilung. 


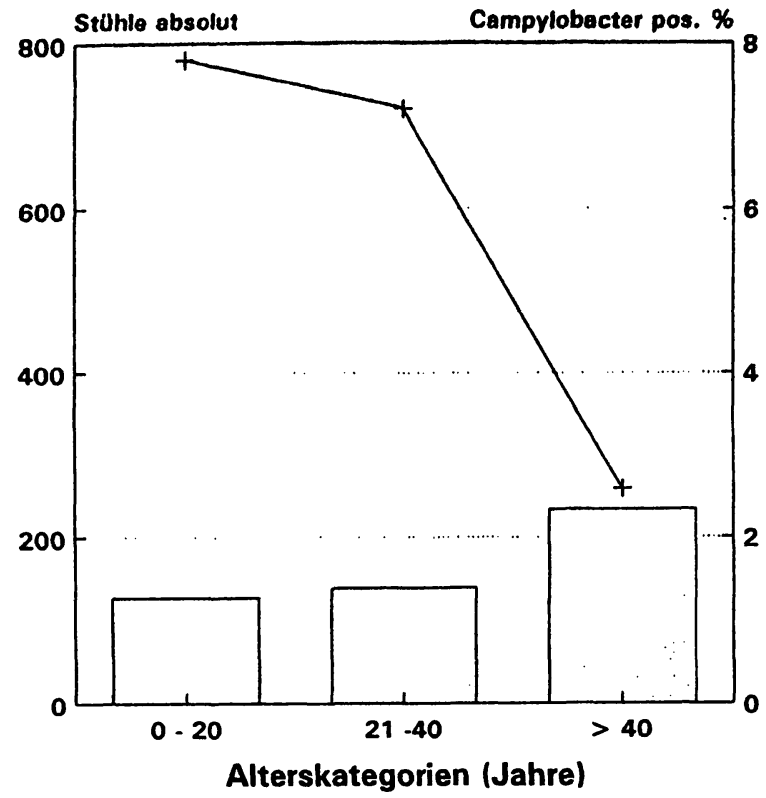

Stūhle absolut $\quad+$ Campylobacter pos \%

Abb. 3: Nachweis von Campylobacter bei Patienten mit Durchfall (Gruppe $b, n=500$ ) - Altersverteilung.

die größte Campylobacter-Auffindungsrate in der mittleren Alterskategorie (21 - 40 Jahre) zu verzeichnen war. Dieser Effekt ist ebenfalls statistisch signifikant $\left(x^{2}=7,02\right.$ $\mathrm{df}=2, \mathrm{p}<0.05)$.

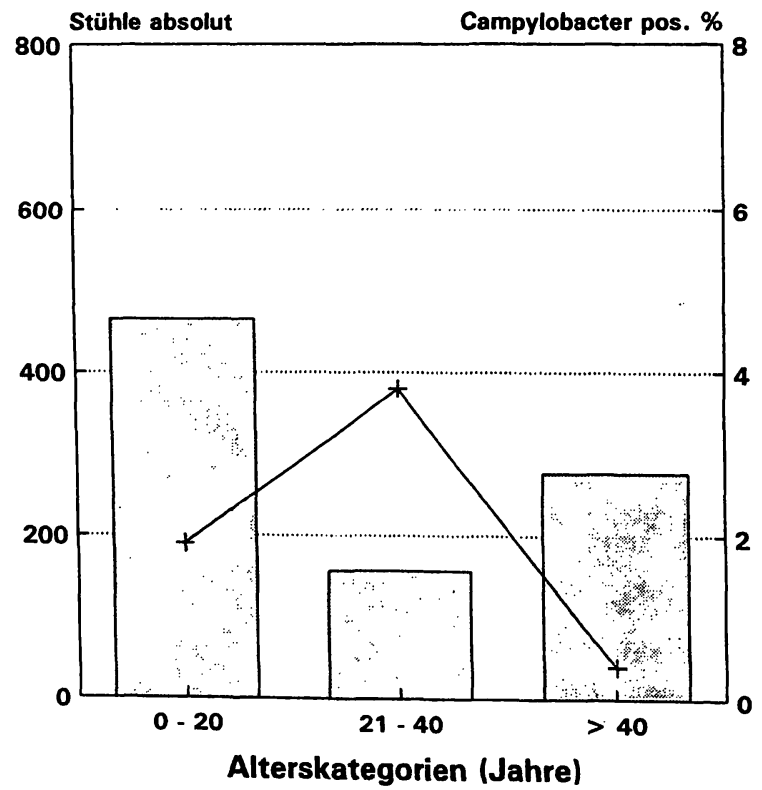

Stühle absolut $\quad+$ Campylobacter pos $\%$

Abb. 4: Nachweis von Campylobacter bei Patienten ohne Durchfall (Gruppe $c, n=900$ ) - Altersverteilung
Unter den 54 isolierten Campylobacter-Stämmen überwogen C. jejuni (41/54 [76\%] und C. coli (9/54 [16,6\%]); in 2 Fällen konnten jeweils $\mathrm{C}$. laridis $(3,7 \%)$ und $\mathrm{C}$. upsaliensis $(3,7 \%)$ nachgewiesen werden.

\section{Diagnostik}

\section{Kultivierungsmethoden:}

Unter den drei Kultivierungsmethoden war das Filterverfahren (Methode 1) dasjenige mit der besten Campylobacter-Ausbeute: Der statistische Prüftest ergab ein $x^{2}$ von 20,15 ( $d f=2, p<0.01$ ), während beim Einzelvergleich der beiden Fest-Selektivmedien (Methode 2 und 3) kein signifikanter Unterschied festgestellt werden konnte (Tabelle 1).

\begin{tabular}{|c|c|c|c|c|}
\hline \multirow[b]{2}{*}{$\begin{array}{l}\text { Isolierte } \\
\text { Campylobacter- } \\
\text { Stämme } \\
\text { gesamt }\end{array}$} & \multicolumn{4}{|c|}{ Kultivierungsmethoden: } \\
\hline & $\begin{array}{l}\text { 1) } \\
\text { Filtermethode }\end{array}$ & $\begin{array}{l}\text { 2) } \\
\text { Blutfreies } \\
\text { Selektivmedium }\end{array}$ & $\begin{array}{l}\text { 3) } \\
\text { Blutmedium } \\
\text { mit Butzler- } \\
\text { Supplement }\end{array}$ & $\begin{array}{l}x^{2} \\
=20,15 \\
(d f=2)\end{array}$ \\
\hline $54(100 \%)$ & $44 / 54(81,5 \%)$ & $22 / 54(50,0 \%)$ & $22 / 54(40,7 \%)$ & $p<0.01$ \\
\hline
\end{tabular}

Tabelle 1: Isolierung von 54 Campylobacter-Stämmen aus 2000 Stuhlproben: Vergleich der drei Kultivierungsmethoden.

In der Abb. 5 wird die Campylobacter-Auffindungsrate durch die drei Kultivierungsmethoden bzw. deren Kom-

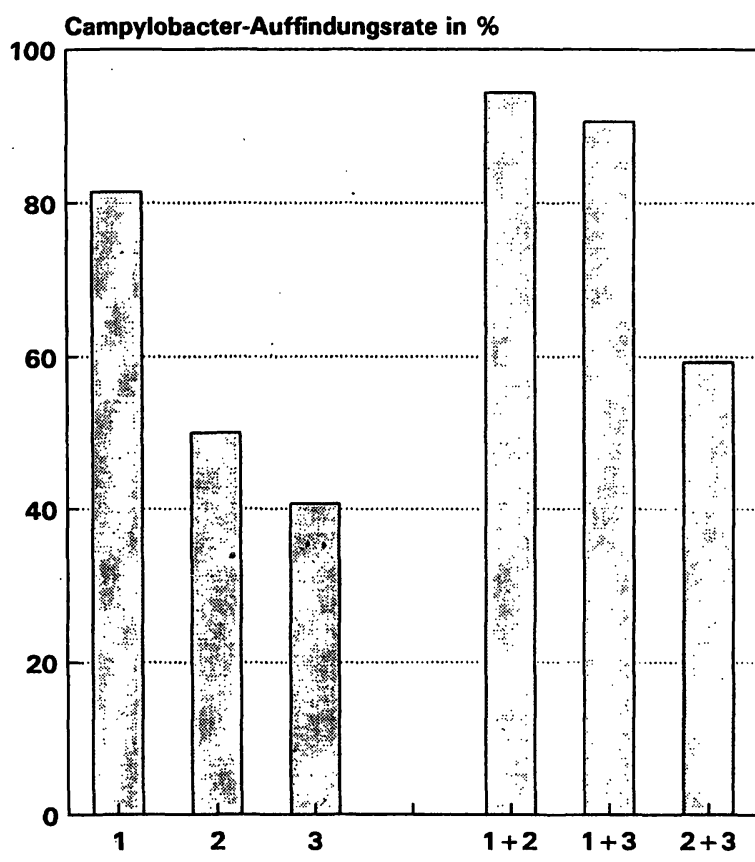

Kultivierungsmethode bzw. Kombinationen

Abb. 5: Campylobacter-Auffindungsrate in \% - Vergleich von drei Kultivierungsmethoden bzw. -kombinationen: 1. Filtrationsverfahren, 2. blutfreier Campylobacter-Selektivagar, 3. Blutmedium mit Butzler-Supplement. 
binationen mittels Balkengraphiken dargestellt. Wie die Augenscheinanalyse zeigt, läßt sich mit Hilfe der Kombinationen Filtermethode + Selektivmedium eine Steigerung der Campylobacter-Ausbeute realisieren, die sich in einem Fall (Methode $1+2$ ) der 100\%-Rate annähert. Diese Steigerungsrate ist signifikant höher $(p<0.05)$ im Vergleich zu einer alleinigen Anwendung der Filtermethode $\left(x^{2}=4,29 \mathrm{df}=1\right)$. Die Kombination von Methode $1+3$ ergibt demgegenüber jedoch keinen signifikanten Unterschied.

In Abb. 6 wird die Aufteilung der 54 isolierten Campylobacter-Stämme auf die entsprechenden Kultivierungsverfahren bzw. deren Korrelation untereinander dargestellt. Daraus geht beispielsweise hervor, daß nur $15 \mathrm{der}$ insgesamt 54 isolierten Stämme $(27,8 \%)$ in allen 3 Kultivierungsverfahren gleichzeitig gefunden wurden. Demgegenüberwurden 22 Stämme nur mit der Filtermethode

\section{Campylobacter-Isolierte Stämme Gesamt $-\mathbf{n}=54$}

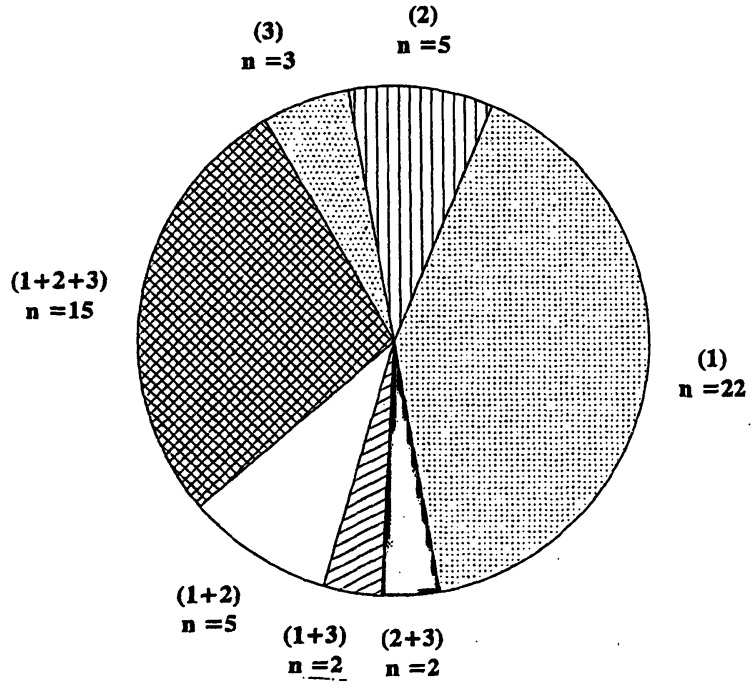

Abb. 6: Isolierung von 54 Campylobacterstämmen laus 2000 Stuhlproben) mit Hilfe von drei parallel eingesetzten Kultivierungsmethoden: 1. Filtrationsverfahren, 2. blutfreier CampylobacterSelektivagar, 3. Blutmedium mit Butzler-Supplement. In Klammern: Kultivierungsmethode bzw. Kombinationen von Kultivierungsmethoden; $n=$ isolierte Stämme.

entdeckt, während 5 Stämme alleine mit dem blutfreien Selektivmedium bzw. 3 Stämme lediglich mit der BlutSelektivplatte erfaßt wurden.

\section{Transportnährboden:}

Der Vergleich von Cary-Blair-Medium versus Nativstuhl $(n=517)$ ergab folgendes Resultat: Es konnten 15 Campylobacter-Stämme aus dem Transportmedium angezüchtet werden gegenüber nur 12 Isolaten aus Nativ-Stühlen. Das entspricht einer um 25\% höheren CampylobacterAusbeute bei Verwendung des Cary-Blair-Transportnährbodens (Tab. 2).

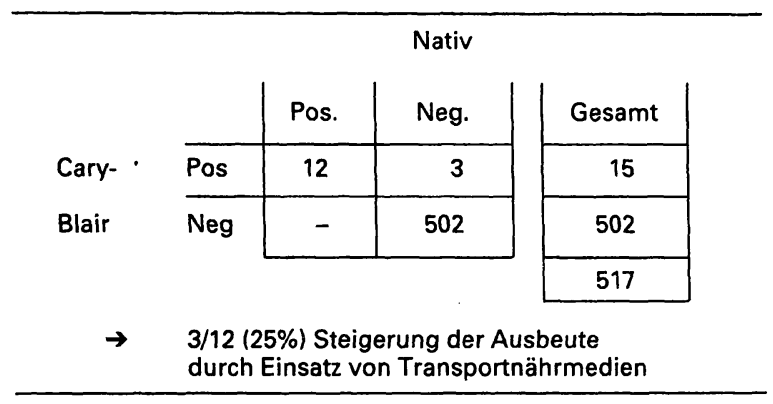

Tabelle 2: Campylobacter-Auffindungsrate beim Vergleich von Transportnährboden (Cary-Blair-Medium) vs. Nativstuhl $(n=517)$.

\section{Diskussion}

Nach Blaser et al. ist Campylobacter jejuni die häufigste Ursache von bakteriell bedingter Gastroenteritis in den industrialisierten Ländern (20). Auf Afrika, Australien, Europa und Nordamerika bezogen soll $C$. jejuni häufiger bei Durchfallpatienten isoliert werden als Salmonellen und Shigellen zusammengenommen (21). In Neuseeland sollen $67 \%$ aller gemeldeten gastrointestinalen $\mathrm{Er}$ krankungen durch Campylobacter-Infektionen bedingt sein (22).

Die Ergebnisse unserer Studie zeigen deutlich, daß Patienten mit Durchfall (Gruppe b) signifikant häufiger Campylobacter-positiv sind $(5,2 \%, A b b .1)$ als Tropenreisende (Gruppe $a-2,0 \%$ ) oder Patienten mit gastrointestinalen Symptomen, jedoch ohne Durchfall (Gruppe $c-1,77 \%)$. Dies läßt den Schluß zu, daß in der Praxis alle dưrchfälligen Stühle routinemäßig neben anderen Erregern auch auf Campylobacter spezies hin untersucht werden sollten.

Ebenso wie von anderen Autoren beschrieben $(2,20,23)$, wurden in unserer Studie am häufigsten C. jejuni (76\%) und C. coli $(16,6 \%)$ isoliert.

Da in den Entwicklungsländern die Prävalenz von Campylobacter-Infektionen durch unzureichende lebensmittelhygienische Bedingungen im Vergleich zu den industrialisierten Ländern um ein Vielfaches höher ist, $(1,2$, 24) hätten wir bei dem Kollektiv der Tropenreisenden eine höhere Infektionshäufigkeit erwartet. In diesem Zusammenhang sei jedoch noch einmal erwähnt, daß die meisten Probanden dieses Kollektivs (insgesamt $n=600$ ) symptomlos waren, lediglich $19(3,2 \%)$ hatten zum Zeitpunkt der Untersuchung Durchfall. Die von diesen 19 Patienten gewonnenen flüssigen Stuhlproben waren allerdings in $15,8 \%(n=3)$ Campylobacter-positiv, was im Vergleich zum Kollektiv der Durchfall-Patienten im Raum Frankfurt $(5,2 \%$ positive Befunde) signifikant höher ist ( $p$ $<0.05$ ). Unsere Untersuchungen an Tropenreisenden mit Durchfall bestätigen somit in der Tendenz die Beobachtung, daß in tropischen Ländern Campylobacter-Infektionen sehr verbreitet sind $(1,2)$.

Bezüglich der Altersverteilung von Campylobacter-Infektionen sind in den industrialisierten Ländern hauptsächlich Kinder und junge Erwachsene betroffen (24 26), wobei die Gründe hierfür bis jetzt nicht eindeutig 
geklärt sind (2). Auch in unserer Studie konnte ein Alterseffekt bei den Patienten mit (Gruppe b) und ohne Durchfall (Gruppe c) beobachtet werden: Patienten < 40 Jahre waren signifikant häufiger mit Campylobacter infiziert als Patienten, die älter als 40 Jahre alt waren (Abb. 3 u. 4). Demgegenüber ist in unseren Untersuchungen bei den Tropenreisenden (Gruppe a) kein Alterseffekt erkennbar.

Unsere Ergebnisse zeigen eine schlechte Korrelation innerhalb der drei geprüften Kultivierungsverfahren. Lediglich 15 der 54 isolierten Stämme $(27,8 \%)$ ließen sich mit allen 3 Methoden nachweisen (Abb. 6). Die restlichen 39 Isolate wurden nur mit einer oder zwei Methoden entdeckt. Übereinstimmend mit anderen Autoren ist daher festzustellen $(15,19,27,28)$, daß bei Anwendung nur eines Kulturverfahrens eine optimale CampylobacterAusbeute nicht gewährleistet werden kann.

Ein Hauptproblem bleibt das mehr oder weniger stark ausgeprägte Überwuchern der Platten mit Enterobakteriazeen der Faekalflora trotz der Antibiotikazusätze. Andererseits können aber auch bestimmte CampylobacterArten (z.B. C. upsaliensis) durch Antibiotika (z.B. Cephalosporine) im Wachstum gehemmt werden.

Eine Möglichkeit, die Kontaminationsprobleme zu umgehen sowie Antibiotika (Cephalosporin)-sensible Campylobacter spp. zu finden, bietet die Filtrationstechnik (18). Diese Technikergab in unserem Labor eine signifikant bessere Ausbeute im Vergleich mit den beiden anderen Isolierungsmethoden ( $81,5 \%$ gegenüber $50 \%$ bzw. $40,7 \%$, vgl. Tab. 1 und Abb. 5). Von insgesamt 54 isolierten Stämmen waren $22(40,7 \%)$ Stämme sogar nur mit dem Filtrationsverfahren nachweisbar (Abb. 6). Unter diesen Isolaten befanden sich auch die zwei $C$. upsaliensisStämme, die durch die Selektivmedien, welche u.a. ein Cephalosporin als Antibiotikazusatz enthielten, nicht hätten entdeckt werden können. Der Vorteil des Filtrationsverfahrens bestand aber vor allem darin, daß durch das Zurückhalten der Faekalflora Kontaminationen praktisch ausgeschlossen wurden. Als Nachteil der Filtermethode ist zu erwähnen, daß es einiger aufwendiger Arbeitsschritte bedarf im Vergleich zum einfachen "handling" beim Beimpfen des Stuhis auf die Selektivmedien. Trotz dieser Nachteile erscheint die Anwendung des Filtrationsverfahrens - allein oder in Kombination mit einem Selektivmedium - für eine maximale Campylobacter-Ausbeute zwingend geboten. Diese Feststellung gilt so lange, bis der Nachweis erbracht werden konnte, daß mit einem Selektivmedium allein gleich gute oder bessere Resultate erreicht werden konnten. Neuere Selektivmedien sind unter diesem Aspekt zu prüfen. In diesem Zusammenhang ist eine Studie von Bolton et al. (1988) von Interesse, in der u.a. zwei Selektivmedien mit der Filtrationsmethode parallel eingesetzt wurden. Hier ergab das Filtrationsverfahren nur $65 \%$ Ausbeute, während mit den beiden anderen Isolierungsmethoden (modifizierter CCD-Agar und Fennell's Medium) $89 \%$ bzw. $86 \%$ Campylobacter spp. isoliert werden konnten (19). Bei einer Bestätigung dieser Befunde wird möglicherweise das Filtrationsverfahren in Zukunft ganz entbehrlich.

Interessanterweise konnte die Campylobacter-Auffindungsrate durch den Einsatz von Cary-Blair-Transportnährmedium um $25 \%$ gesteigert werden.

Folgende Schlußfolgerungen ziehen wir aus unseren Ergebnissen und der vorliegenden Literatur: Durchfällige Stuhlproben sollten prinzipiell auch auf Campylobacter spp. hin untersucht werden. Nach Möglichkeit sollte bei allen Untersuchungen eine Kombination zweierverschiedener Anzuchtmedien zur Anwendung kommen. Bei Prüfung neuer Selektivmedien ist es derzeit noch empfehlenswert, das antibiotikafreie Filterverfahren (18) in den Vergleich mit einzubeziehen. Schließlich ist in besonderen Fällen (z.B. langer Probentransport) an die Verwendung eines Transport-Nährmediums zu denken.

\section{Danksagung:}

Wir danken Frau Dr. med. U. Mikulicz, Tropenmedizinische Untersuchungsstelle der GTZ, Eschborn/Ts. und Herrn Dr. med. H. Koeppen, Kinderklinik des Städtischen Krankenhauses Frankfurt/ M.-Höchst für die kooperative Zusammenarbeit bezüglich der Untersuchungen mit dem Transportnährmedium. 
Literatur:

1. Kist, M. (1992): Schraubenbakterien mit starrer Zellwand. In: Mikrobiologische Diagnostik (Burkardt, F., ed). Georg Thieme Verlag Stuttgart - New York. pp. $112-118$.

2. Skirrow, M.B. (1990): Foodborne Illness: Lancet, Vol. 336, 921 - 923.

3. WHO (1980): Enteric infections due to Campylobacter, Yersina, Salmonella and Shigella. Bull. WHO 58, 519-537.

4. Gerigk, K., Teufel, P. (1990): Lebensmittelinfektionen und -intoxikationen. Bundesgesundhbl. 3/90, $89-93$.

5. Balacescu, C., Onöz, E., Hensel, G., van Treeck, U., Hüllen, F.U., Höffler, U. (1986): Laboruntersuchungen auf bakterielle enterale Infektionen im Bereich Nordrhein von 1966 bis 1985 . Öff. Gesund.-Wes 48, $336-337$.

6. Smith. T. (1918): Spirilla associated with disease of the fetal membranes in cattle (infectious abortion). J. exp. Med. 28, $701-719$.

7.Smith, T., Taylor, M.S. (1919): Some morphological and biological characters of the spirilla (Vibrio fetus n.sp.) associated with disease of the fetal membranes in cattle. J. exp. Med. 30, 299-311.

8. Jones, F.S., Orcutt, M., Little, R.B. (1931): Vibrio associated with intestinal disorder of cows and calves. J. exp. Med. 53, 853.

9. Dekeyser, P., Gossoin-Detrain, M., Butzler, J.P., Sternon, J. (1972): Acute enteritis due to related Vibrio: first positive stool culture. J. infect. Dis. 125, $390-392$.

10. Skirrow, M.B. (1974): Campylobacter enteritis: a "new" disease. Brit. med. J. 2, 9-11.

11. Butzler, J.P., Skirrow, M.B. (1979): Campylobacter enteritis. Clin. Gastroenterol. 8, $737-765$.

12. Blaser, M.J., Berkowitz, I.D., LaForce, F.M., Cravens, J., Reller, L.B., Wang, W.I. (1979): Campylobacter enteritis: clinical and epidemiological features. Ann. Intern. Med. 91, $179-185$.

13. Bolton, F.J., Robertson, L. (1982): A selective medium for isolating Campylobacter jejuni/coli. J. Clin. Pathol. 35, 462 - 467.

14. Goossens, H., De Boeck, M., Skirrow, M.B. (1983): A new selective medium for the isolation of Campylobacter jejuni from human feces. Eur. J. Clin. Microbiol. 2, $389-394$.

15. Karmali, M.A., Simor, A.E., Roscoe, M., Fleming, P.C., Smith, S.S., Lane J. (1986): Evaluation of a blood-free, charcoal-based, selective medium for the isolation of Campylobacter organišms from feces. J. Clin. Micobiol. 23, $456-459$.

16. Goossens, H., Vlaes, L., De Boeck, M., Pot, B., Kerster, K., Levy, J., De Mol, P., Butzler, J.P., Vandamme, P. (1990): Is "Campylobacter upsaliensis" an unrecognised cause of human diarrhoea? Lancet 335, $584-586$.

17. Cary, S.G., Blair, E.B. (1964): New transport medium for shipment of clinical specimens. J. Bacteriol. 88, 96-98.
18. Steele, T.W., McDermott, S.N. (1984): The use of membrane filters applied directly to the surface of agar plates for the isolation of Campylobacter jejuni from feces. Pathology 16, 263- 265.

19. Bolton, F.J., Hutchinson, D.N., Parker, G. (1988): Reassessment of selective agars and filtration techniques for isolation of Campylobacter species from faeces. Eur. J. Clin. Microbiol. Infect. Dis., 7, 155 - 160.

20.Blaser, M.J., Hopkins, J.A., Vasil, M.L. (1984): Campylobacter enteritis. N. Engl. J. Med. 305, $1444-1452$.

21. Blaser, M.J., Wells, J.G., Feldman, R.A., Pollard, R.A., Allen, J.R., the Collaborative Diarrhea Disease Study Group (1983): Campylobacter enteritis in the United States: a multicenter study. Ann. Intern. Med. 98, 360 - 365.

22. New Zealand Communicable Disease Centre. Communicable Disease New Zealand (1989): Annual supplement. Porirua, New Zealand, 16.

23. Butzler, J.-P., Goossens, H. (1988): Campylobacter jejuni infection as a hospital problem: an overview. Jorunal of Hospital Infection 11, Suppl. A, $374-377$.

24. Brandis, H. (1988): Die Gattungen Streptobacillus und Campylobacter. In: Lehrbuch der medizinischen Mikrobiologie (Brandis, H. u. Pulverer, G., eds.). Gustav Fischer Verlag Stuttgart - New York. pp. 406-410.

25. Blaser, M.J., Taylor, D.N., Feldman, R.A. (1983): Epidemiology of Campylobacter jejuni infections. Epidemiol. Rev. 5, 157 - 176.

26. Skirrow, M.B. (1987): A demographic survey of campylobacter, salmonella and shigella infections in England. A Public Health Laboratory Service Survey. Epidemiol. Infect. 99, 647 - 657

27. Bolton, F.J., Coates, D., Hinchcliffe, P.M., Robertson, L. (1983): Comparison of selective media for isolation of Campylobacter jejuni/coli. J. Clin. Pathol. $36,78-83$.

28. Patton, C.M., Mitchell, S.W., Potter, M.E., Kaufmann, A.F. (1981): Comparison of selective media for primary isolation of Campylobacter fetus subsp. jejuni. J. Clin. Microbiol. 13, 326 - 330.

\section{Anschriften der Verfasser:}

Dr. med. Sabine Albert

Sabine Bachmann

Prof. Dr. med. Volker Brade

Abteilung Medizinische Mikrobiologie

im Zentrum der Hygiene,

Klinikum der J.W. Goethe-Universität Frankfurt/M.

Paul-Ehrlich-Str. 40

60596 Frankfurt/M. 